OPEN ACCESS

Edited by:

Raul Antonio Sperotto, University of Taquari Valley, Brazil

Reviewed by:

R. J. Neil Emery,

Trent University, Canada

Concepción Gómez-Mena,

Instituto de Biología Moleculary

Celular de Plantas (IBMCP), Spain

*Correspondence:

Elena A. Dolgikh

dol2helen@yahoo.com

Specialty section:

This article was submitted to

Plant Microbe Interactions,

a section of the journal

Frontiers in Plant Science

Received: 04 December 2018

Accepted: 21 March 2019

Published: 09 April 2019

Citation:

Dolgikh AV, Kirienko AN, Tikhonovich IA, Foo E and Dolgikh EA (2019) The DELLA Proteins Influence the Expression of Cytokinin Biosynthesis and Response Genes

During Nodulation.

Front. Plant Sci. 10:432.

doi: $10.3389 /$ fpls.2019.00432

\section{The DELLA Proteins Influence the Expression of Cytokinin Biosynthesis and Response Genes During Nodulation}

\author{
Alexandra V. Dolgikh ${ }^{1}$, Anna N. Kirienko', Igor A. Tikhonovich', Eloise Foo ${ }^{2}$ and \\ Elena A. Dolgikh ${ }^{1 *}$ \\ ${ }^{1}$ All-Russia Research Institute for Agricultural Microbiology, St. Petersburg, Russia, ${ }^{2}$ School of Natural Sciences, University \\ of Tasmania, Hobart, TAS, Australia
}

The key event that initiates nodule organogenesis is the perception of bacterial signal molecules, the Nod factors, triggering a complex of responses in epidermal and cortical cells of the root. The Nod factor signaling pathway interacts with plant hormones, including cytokinins and gibberellins. Activation of cytokinin signaling through the homeodomain-containing transcription factors KNOX is essential for nodule formation. The main regulators of gibberellin signaling, the DELLA proteins are also involved in regulation of nodule formation. However, the interaction between the cytokinin and gibberellin signaling pathways is not fully understood. Here, we show in Pisum sativum L. that the DELLA proteins can activate the expression of KNOX and BELL transcription factors involved in regulation of cytokinin metabolic and response genes. Consistently, pea la cry-s (della1 della2) mutant showed reduced ability to upregulate expression of some cytokinin metabolic genes during nodulation. Our results suggest that DELLA proteins may regulate cytokinin metabolism upon nodulation.

Keywords: legume-rhizobium symbiosis, root nodule development, mutants, cytokinins, gibberelllins, crosstalk

\section{INTRODUCTION}

Most legume plants are able to form symbiosis with nitrogen-fixing bacteria that results in a new organ formation, the nodule. Multiple studies have shown that root nodule formation is regulated by several plant hormones (reviewed by Ferguson and Mathesius, 2014; Gamas et al., 2017). However, the mechanisms of this regulatory network remain poorly understood. The essential role of cytokinins (CKs) in symbiosis development and their interplay with Nod factor-activated symbiotic signaling pathway has been shown. Indeed, in loss-of-function Ljlhk1 and Mtcre1 mutants defective in the receptors to CKs, a significant decrease in the number of nodules was found (Gonzalez-Rizzo et al., 2006; Murray et al., 2006; Plet et al., 2011). It was also shown that gain-of-function mutants in genes encoding receptors to CKs have an ability to form spontaneous nodule structures in the absence of compatible bacteria and exogenous application of CKs mimics the effect of Nod factor treatment in root cortex (Cooper and Long, 1994; Tirichine et al., 2007; Heckmann et al., 2011; Ovchinnikova et al., 2011). At the same time it has been found that CKs can play both positive and negative

Abbreviations: IPT, isopentenyl transferase; LOG, lonely guy; qRT-PCR, quantitative reverse transcription PCR; $R R$, response regulator; $\mathrm{Y} 2 \mathrm{H}$, yeast two hybrid system. 
role in symbiosis depending on place, time and surrounding phytohormonal network (Gamas et al., 2017). This means that CK-activated signaling must be tightly regulated during nodulation and may interact with other phytohormonal signaling pathways during nodulation.

The gibberellins (GAs) are well-known phytohormones control many aspects of plant growth and development (Brian, 1959; Harberd et al., 1998). GAs have also been shown to play an important role during nodulation. Indeed, application and genetic studies indicate GAs can have both positive and negative effects of the number of nodules formed (e.g., Ferguson, 2005, Ferguson et al., 2011; Maekawa et al., 2009; Fonouni-Farde et al., 2016; Jin et al., 2016; McAdam et al., 2018). Like CKs, GAs appear to act at various stages of infection and nodule development (McAdam et al., 2018). Considering the functions of GAs and CKs it is possible these hormones interact to influence nodule initiation and development.

The active forms of GAs are perceived by the GID1 (GA insensitive dwarf1) receptor that in turn binds to and activates the degradation of DELLA proteins, via $26 \mathrm{~S}$ proteasome (Alvey and Harberd, 2005; Achard et al., 2006, 2007; Navarro et al., 2008). The DELLAs are transcriptional regulators that repress GA responses and thus the DELLA may be considered as negative regulators of GA-signaling (Sun, 2010; Hedden and Thomas, 2012). As a consequence, the level of DELLA proteins can be changed depending on different external and internal factors (Davière and Achard, 2013; Park et al., 2013). Indeed, DELLA proteins may mediate crosstalk with other hormonal signaling pathways, coordinating the plants growth with responses to various stimuli (Floss et al., 2016; Fonouni-Farde et al., 2017).

It is known that DELLA proteins do not have DNAbinding domain and their regulatory ability is based on the interaction with different transcription factors, whose activity can be stimulated or inhibited in such interactions (Sun and Gubler, 2004; Fleet and Sun, 2005). Previous studies in legume plants have shown that DELLA proteins may interact with some transcriptional regulators in Nod factor-activated signaling pathway and modulate their function (Floss et al., 2016; Fonouni-Farde et al., 2016; Jin et al., 2016). Indeed, an important role for DELLA proteins in nodulation is supported by low infection and nodule number observed in della mutants or loss-of-function transgenics across legume species (Fonouni-Farde et al., 2016; Jin et al., 2016; McAdam et al., 2018). This may be mediated by physical interaction of the DELLA proteins with key components of the Nod factor signaling pathway including IPD3/CYCLOPS, NSP2 and NF-YA1 (Fonouni-Farde et al., 2016; Jin et al., 2016). However, it is important to note that a positive role for GA in nodule organogenesis has also been reported, as severley GA-deficient mutants of pea form many infections but few nodules that are poorly developed (McAdam et al., 2018). In contrast, the few nodules that form on della mutants of pea and Medicago appear to be normal size and at least in pea have been shown to have similar function to wild type (McAdam et al., 2018).
In order to find possible mechanisms of interplay between GA- and CK-signaling pathways, we have examined how the main regulators of the GA-signaling, the DELLA proteins, may influence KNOTTED1-LIKE HOMEOBOX (KNOX) and BEL1-LIKE HOMEODOMAIN (BELL) transcription factors involved in control of CKs and GAs levels in plants. Plant KNOX and BELL proteins are transcription factors that belong to the three-amino-acid-loop-extension (TALE) superfamily (Byrne et al., 2003; Smith and Hake, 2003; Bao et al., 2004; Sharma et al., 2014). These transcription factors interact as heterodimeric complexes to regulate transcription of target genes (Bellaoui et al., 2001; Chen et al., 2004; Hake et al., 2004; Lin et al., 2013). It is known that KNOX transcription factors may control the levels of CKs and GAs via direct regulating of CK biosynthesis IPT gene (Yanai et al., 2005) as well as GA metabolic genes, such as GA 20-OXIDASE (GA20ox) and GA 2-OXIDASE (GA2ox) in shoot apical meristem (Jasinski et al., 2005). Moreover, we have recently reported that KNOX3 transcription factor may promote the expression of some IPT and LOG genes during symbiosis development in legume plants M. truncatula and pea Pisum sativum L. (Azarakhsh et al., 2015). Recent study has also revealed that GA signaling mediated by MtDELLA1 decreases the amount of the free base CK content in roots of $M$. truncatula (Fonouni-Farde et al., 2017). However, the interaction between CKs and GAs during nodulation is still not known and the mechanisms of DELLAs' impact on CKs status remain poorly understood.

Here we used the pea La cry-s (DELLA1 della2) and la cry-s (della1 della2) mutants to examine the effects of DELLA proteins on KNOX and BELL transcription factors. We have found that the up-regulation of expression of some $P s K N O X$ and PsBELL genes seen in wild type was reduced in double la cry-s (della1 della2) mutant during initial and later stages of nodulation. Pea la cry-s (della1 della2) mutants also showed reduced capacity to elevate expression of the CK metabolic and signaling genes during nodulation compared to wild type. Therefore, PsDELLA proteins may be involved in regulation of the $\mathrm{CK}$ metabolism during nodulation. To test if this action of DELLA was via KNOX and BELL transcription factors, the $\mathrm{Y} 2 \mathrm{H}$ studies were undertaken but did not reveal a strong direct interaction between PsDELLA1 and PsKNOX3 or PsBELL1. Therefore, PsDELLA1 may promote the expression PsKNOX3 and PsBELL1 and their target CK metabolic and signaling genes indirectly through other known transcription factors and we begin to examine the role of PsIPD3/CYCLOPS.

\section{MATERIALS AND METHODS}

\section{Bacterial Strains and Inoculation}

Inoculation of the pea plants was conducted with the Rhizobium leguminosarum biovar viciae wild type strain 3841. Bacterial liquid culture was grown in $\mathrm{B}^{-}$medium (van Brussel et al., 1977), diluted upto the optical density at $600 \mathrm{~nm}\left(\mathrm{OD}_{600}\right) 0.5$ and applied to plants at 2 day after planting. 


\section{Plant Material and Growth Conditions}

The pea Pisum sativum L. La cry mutant lines and line segregating la cry carrying the mutations in della 1 and della 2 genes were generated as described by Weston et al. (2008) and wild type line is cv. Torsdag. Wild type SGE line and two derived mutants SGEFix ${ }^{-}-2$ and SGEFix ${ }^{-}-5$ [ipd3/cyclops (sym33)] were also used in this study. Gene expression experiments were carried out with seeds sterilized with sulphuric acid for $5 \mathrm{~min}$, washed 3 times with water, transferred on $1 \%$ water agar plates and germinated at room temperature in the dark. 4-5 days-old plant seedlings were transferred into pots with vermiculite saturated with Jensen medium (van Brussel et al., 1982), grown in a growth chamber at $21^{\circ} \mathrm{C}$ at $16 \mathrm{~h}$ light $/ 8 \mathrm{~h}$ dark cycles, $60 \%$ humidity. Fragments of main roots (responsive zone starting from 5 to $6 \mathrm{~mm}$ from the root tip) or fragments of main roots with primordia/nodules were collected at 2, 9, and 14 days after inoculation (2, 9, and 14 dai). Fragments of non-inoculated main roots were collected at the same developmental stages. Plant material for gene expression studies was immediately immersed in liquid nitrogen and stored in $-80^{\circ} \mathrm{C}$ freezer.

\section{RNA Extraction, cDNA Synthesis, and Quantitative PCR}

Plant material was ground with a mortar and pestle to a fine powder in liquid nitrogen. Approximately 50-100 mg of ground tissue was used for RNA extraction, as previously described (Dolgikh et al., 2017). 1-2.5 $\mu \mathrm{g}$ of total RNA was used to synthesize cDNA with the RevertAid Reverse Transcriptase (Thermo Fisher Scientific, United States). cDNA samples were diluted to a total volume of $200 \mu \mathrm{l}$. For gene expression quantification, the following primer pairs were used (Supplementary Table S1). All primers were acquired from Evrogen company ${ }^{1} .2 \mu l$ of cDNA was used for quantitative real-time PCR using Bio-Rad iQ Sybr master mix (BioRad Laboratories, United States) following the manufacturer's recommendations and run on a CFX-96 real-time PCR detection system with C1000 thermal cycler (Bio-Rad Laboratories). All reactions were done in triplicate and averaged. Cycle threshold (Ct) values were obtained using the accompanying software and data were analyzed according to the $2^{-\Delta \Delta \mathrm{Ct}}$ method (Livak and Schmittgen, 2001). All primer pairs (Supplementary Table S2) were designed using the Vector NTI program and produced by Evrogen ${ }^{2}$. The gene expression was normalized against the constitutively expressed ubiquitin gene in pea.The gene expression in experiments with wild type and mutants was determined with several biological samples $(n=4-8$ plants). For temporal BELL1 gene expression, each replicate contained tissue of 3-4 plants and experiment was repeated three times.

\section{Cloning of PsDELLA1, PsKNOX3, and PsBELL1 for Yeast Transformation}

Full-length PsDELLA1, PsKNOX3, and PsBELL1 coding sequences were obtained by amplification of cDNA cv. Finale or cv. Sparkle using specific PCR primer pairs flanking with attB1 and attB2 sequences or CACC in forward primer (Supplementary Table S2) for subsequent cloning in pDONR221 or pENTRY-TOPO vectors (Thermo Fisher scientific, United States) according to manufacturer's protocol.

\footnotetext{
${ }^{1}$ www.evrogen.com

${ }^{2}$ http://www.evrogen.com
}

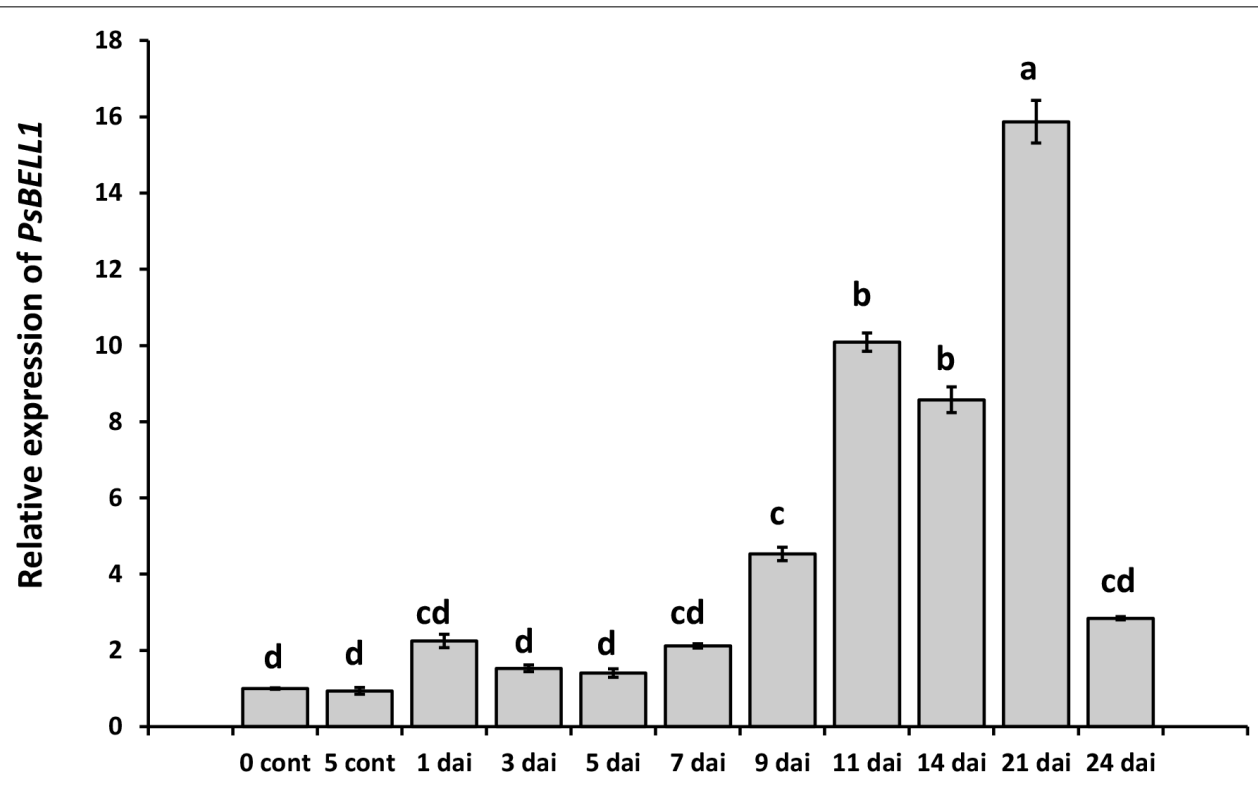

FIGURE 1 | Analysis of PSBELL1 expression at the various stages of symbiosis development in cv. Torsdag. The roots of non-inoculated plants (NI) have been used as a control. The relative expression was normalized against the constitutively expressed ubiquitin and actin genes. Data are averages \pm SEM of three technical repeats. The graphs show the results of one biological experiment, representative for three independent experiments. Values with different letters are significantly different $(P<0.05)$ as analyzed by one-way ANOVA and the Tukey's test as post-hoc analysis. 
At the next stage they were finally subcloned into the destination vectors pDEST22 (PREY) or pDEST32 (BAIT) vectors using the LR clonase enzyme (Thermo Fisher scientific). All verified constructs were transferred into MaV203 yeast strain (Thermo Fisher scientific).

Full-length PSNSP2 and PSIPD3/CYCLOPS coding sequences or partial coding sequences upto stop-codon corresponding to those in RisNod14, E69 nsp2 (sym7) or SGEFix ${ }^{-}-5$ [ipd3/cyclops (sym33)] mutants were obtained by amplification of cDNA cv. Finale or Sparkle using specific PCR primer pairs flanking with attB1 and attB2 sequences followed by cloning into pDEST22 (PREY) or pDEST32 (BAIT) vectors.

\section{Yeast Two-Hybrid Assay (GAL4 Transcription Factor-Based Assay)}

The S. cerevisiae strain MaV203 (Thermo Fisher scientific) was transformed simultaneously with pDEST22 and pDEST32 vectors for GAL4 based selection. To transform the $S$. cerevisiae $\mathrm{MaV} 203$, the protocol for preparation of chemically competent cells was used (Thermo Fisher scientific). A few pairs of vectors (pEXP32/Krev1 and pEXP22/RalGDS-wild type, pEXP22/RalGDS-m1 and pEXP22/RalGDS-m2) suggested by the manufacturer as controls were used for strong, weak and not detectable interactions. Analysis of interaction was conducted on selective media like SC -LT (without leucine and tryptophan), SC -LTH + 3AT (without leucine, tryptophan and histidine plus 3-amino-1,2,4-triazole, SC -LTU (without leucine, tryptophan and uracil).

\section{Statistical Methods and Computer Software}

Multiple alignment of nucleotide sequences was performed using Clustal W (Thompson et al., 1994) using Vector NTI Advance $10\left(\operatorname{InforMax}^{3}\right)$. MEGA6 was used to generate graphic output of phylogenetic tree (Hall, 2013). One-way ANOVA and Tukey's test were used to compare gene expression levels.

\section{RESULTS}

\section{Transcription Levels of PsKNOX and PsBELL Genes in Pea La cry-s (DELLA1 della2) and la cry-s (della1 della2) Mutants}

Previously nine PsKNOX genes were identified in pea $P$. sativum L. (Hofer et al., 2001; Zhou et al., 2014; Azarakhsh et al., 2015). Their expression pattern was estimated during nodulation and showed stimulation of PsKNOX3,PsKNOX5, PsKNOX9, and $P s K N O X 10$ genes upon nodule development starting from 7 to 9 days after inoculation (7-9 dai) (Azarakhsh et al., 2015).

The screening of Mt4.0v1 database for model legume M. truncatula allowed identifying eleven MtBELL genes and eleven homologous genes have been found in pea database ${ }^{4}$

${ }^{3} \mathrm{http}: / /$ www.informaxinc.com

${ }^{4}$ http://bios.dijon.inra.fr
(Supplementary Table S1 and Figure S1). Expression atlas showed that at least one gene PsCam048179 (PsBELL1, the closest homologue of Medtr8g078480) is significantly induced in the nodules compared to roots. Indeed, in our experiments the enhanced level of PsBELL1 has been revealed during nodulation in wild type pea plants of cv. Torsdag (Figure 1). In addition, analysis of transcriptome data for model legume $M$. truncatula showed the activation of some MtKNOX and MtBELL genes in roots in response to Nod factor treatment (van Zeijl et al., 2015; Jardinaud et al., 2016). It suggests that KNOX and BELL transcription factors may be also involved in regulation of early stages of symbiosis development in legumes.

To investigate whether the PsDELLA proteins are involved in regulation of PsKNOX3, PsKNOX5, PsKNOX9, PsKNOX10, and PsBELL1 transcription factors, the transcription levels of corresponding genes have been analyzed in LA cry-s (DELLA1 della2) and la cry-s (della1 della2) mutants in response to inoculation using real-time PCR. The La cry-s (DELLA1 della2) single mutant after inoculation with $R$. leguminosarum bv. viciae showed a nodulation phenotype that did not differ from that of the wild-type, however, the la cry-s (della1 della2) double mutant formed fewer nodules relative to the wild-type but the nodules that formed on mutant plants appeared be functional and to fix similar amount of nitrogen per gram nodule weight (Ferguson et al., 2011; McAdam et al., 2018).

At the early stages of symbiosis development the stimulation of two pea PsKNOX and PsBELL genes, the PsKNOX9 and PsBELL1, was revealed in wild type and LA cry-s (DELLA1 della2) mutant roots in response to inoculation (2 dai) (Figure 2). In contrast, analysis of mutants showed the PsKNOX9 and PsBELL1 expression was not significantly elevated in inoculated la cry-s (della1 della2) mutant (Figure 2). This suggests that PsKNOX9 and PSBELL1 may be involved in the control of early steps of symbiosis in pea and the PsDELLA1 and PsDELLA2 proteins may directly or indirectly influence their activation. Since the effect was more pronounced, when both della1 della2 genes were impaired, it may indicate that the PsDELLA1 could play a more significant role in symbiosis regulation. At the same time it also could be connected with the functional redundancy of PsDELLA1 and PsDELLA2 proteins in pea.

To verify the impact of DELLA proteins on later stages of nodulation, which includes infection thread formation and growth, nodule primordia initiation and development, we have performed the analysis with wild type and mutants in 9 and 14 dai (Figures 3, 4). As previously reported (Azarakhsh et al., 2015), it was also found that PsKNOX3, PsKNOX5, PsKNOX9, and PsKNOX10 expression was upregulated in wild type pea plants in the process of nodulation. In contrast to wild type, there was only little increase in PsKNOX3 and PsKNOX9 expression in la cry-s (della1 della2) mutant in 9 and 14 dai, while the PsKNOX5 showed a moderate upregulation in double la cry-s (della1 della2) mutant in 14 dai (Figures 3, 4). At the same time, the up-regulation of PsKNOX10 expression seen in wild type plants 14 dai was also observed in la cry-s (della1 della2) mutants (Figure 4). Therefore, our study identified PsKNOX3 and PsKNOX9 transcription as potentially regulated by PsDELLA1 and PsDELLA2 proteins 


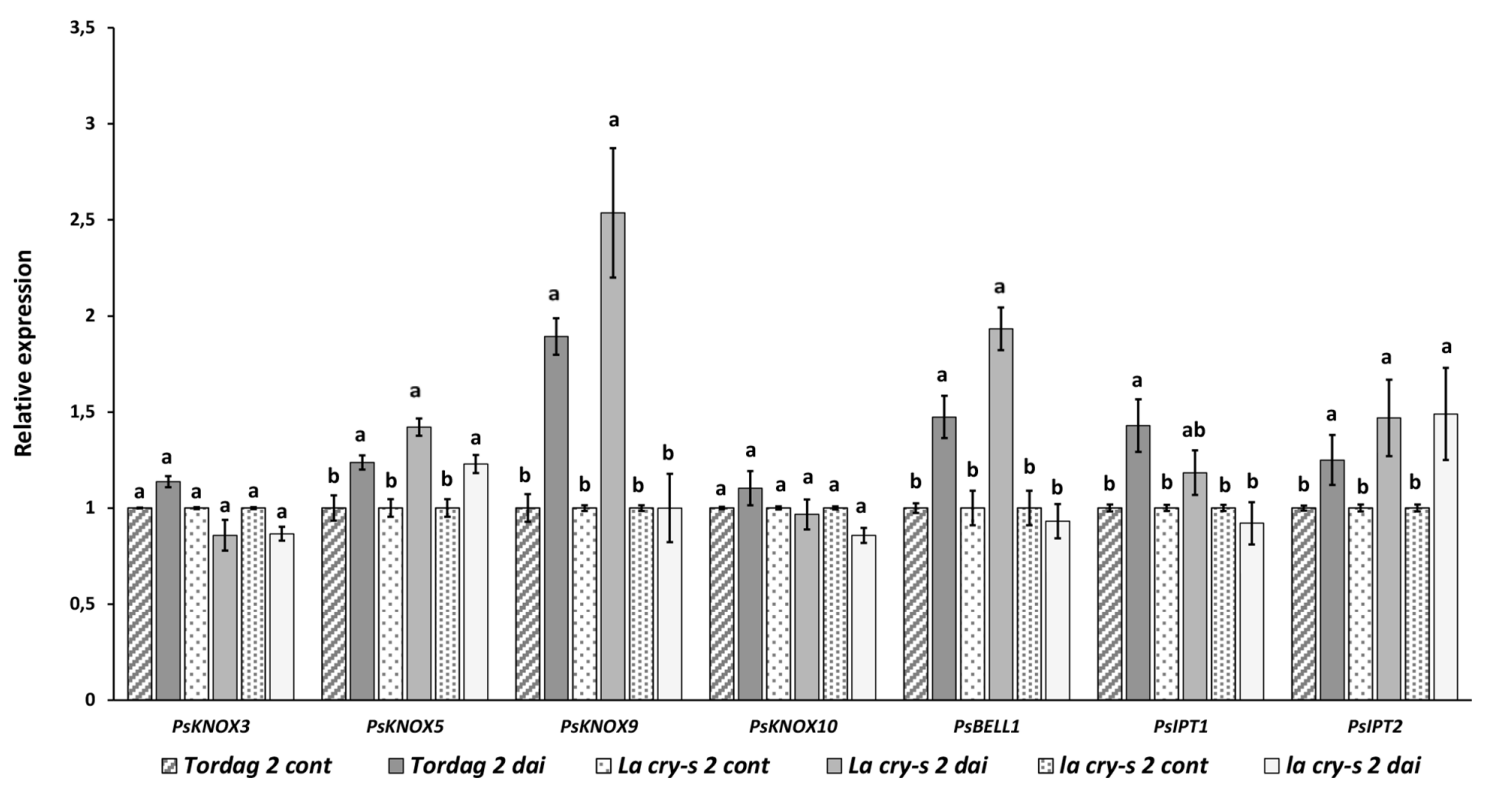

FIGURE 2 | Expression levels of PSKNOX and PSBELL genes and cytokinin metabolic genes in pea wild type cv. Torsdag and LA cry-s (DELLA1 della2), la cry-s (della1 della2) mutants in 2 days after inoculation (2 dai). The expression was normalized against the constitutively expressed ubiquitin gene. For each gene, the transcript level in non-inoculated roots of wild type or mutants was set to 1 (control), and the level in inoculated wild type or mutants was calculated relative to the control values. Data are averages \pm SEM ( $n=5-6$ plants of wild type or mutant combined from two independent experiments). Values with different letters are significantly different $(P<0.05)$ as analyzed by one-way ANOVA and the Tukey's test as post-hoc analysis.

in pea $P$. sativum L. during nodulation. The PsDELLA1 and PsDELLA2 proteins may also be involved in an additional stimulation of the PsKNOX5 expression in pea plants. Similarly, the significant upregulation in the expression of PsBELL1 seen 9 and 14 dai in wild type was not significantly elevated in la cry-s (della1 della2) mutant in response to inoculation (Figures 3, 4). It suggests that PsDELLA1 and PsDELLA2 proteins may also promote the expression of PsBELL1 gene in pea.

\section{Regulation of PsLOG and PsIPT Genes in Pea LA cry and la cry Mutants}

It was shown that expression of some PsLOG and PsIPT genes may depend on the PsKNOX3 transcription factor in pea (Azarakhsh et al., 2015). To investigate a possible link between regulation of transcription factors by DELLA and possible targets of KNOX transcription factors, we have examined the PsLOG1, PsLOG2, PsIPT1, PsIPT2, PsIPT3, and PsIPT4 (all of them encode enzymes that belong to adenosine phosphate-IPTs) expression in LA cry and la cry mutants during nodulation (2, 9, and 14 dai) (Figures 3, 4), because their up-regulation was shown upon nodulation in pea (Azarakhsh et al., 2015; Dolgikh et al., 2017). However, as the level of IPT3 was very low in cv. Torsdag and both mutants it was not included in the analysis. In addition, we have also analyzed the transcription levels of the cytokinin-responsive PsRR11 gene, which is most significantly activated in pea root in response to inoculation in comparison with other PsRRs like PsRR4, PsRR5, PsRR8, and PsRR9 (Dolgikh et al., 2017).
Early stages of symbiosis development may be connected with stimulation of PsIPT1 (the closest homolog of Medtr4g117330) and PsIPT2 (the closest homolog of Medtr2g022140) genes in pea (Dolgikh et al., 2017). However, we did not find essential differences in expression of these genes between wild type $\mathrm{cv}$. Torsdag and LA cry and la cry mutants in 2 dai (Figure 2). As it was shown previously, up-regulation of the PsLOG1, PsLOG2 and PsIPT1, PsIPT3 and PsIPT4 genes involved in CK biosynthesis may take place at later stages of symbiosis development (Azarakhsh et al., 2015; Dolgikh et al., 2017). Our experiments showed that in contrast to stimulation in cv. Tosdag (wild type), the upregulation in expression of the PsLOG1, PsLOG2 genes and cytokinin-responsive PsRR11 genes observed in wild type plants was not observed in the double mutant la cry at 9 and 14 dai, suggesting that PsDELLA1 and PsDELLA2 proteins might be required for this upregulation. The pattern expression of PSIPT1 and PsIPT4 (the closest homolog of Medtrlg110590) expression in response to inoculation was similar in wild type, LA cry and la cry mutants in 9 and 14 dai (Figure 4), suggesting PsDELLA is not essential for the regulation of these genes. A lack of correlation between the change in expression of PsKNOX3 gene and the PsIPT4 gene was previously shown in our experiments (Azarakhsh et al., 2015).

\section{DELLA Proteins Are Able to Regulate GA Metabolic Genes}

It was previously suggested that PsDELLA1 and PsDELLA2 proteins may regulate GA metabolism modulating the expression of corresponding genes in pea (Weston et al., 2008). Indeed, 

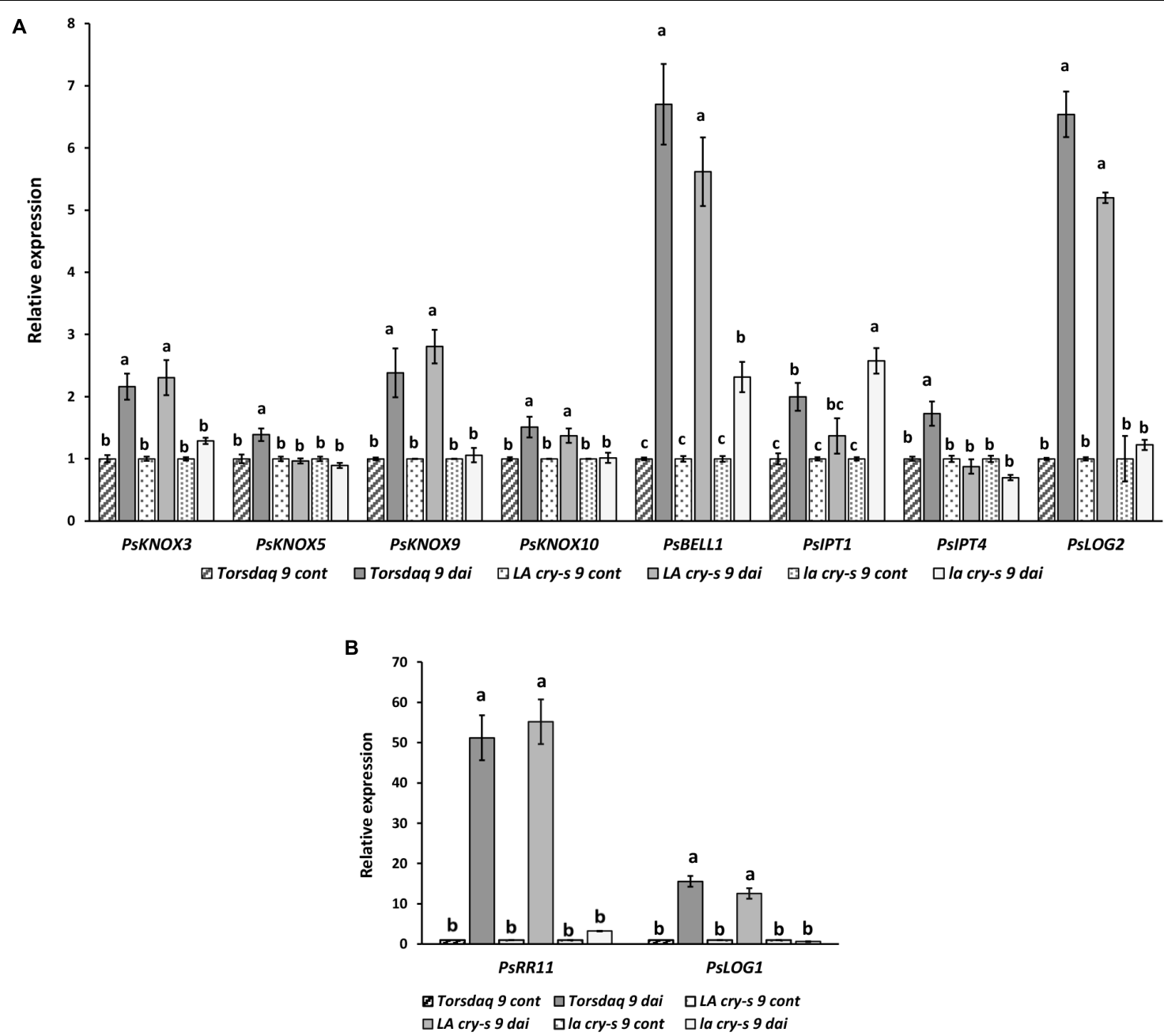

FIGURE 3 | Expression levels of PSKNOX and PSBELL genes and cytokinin metabolic and response genes in pea wild type cv. Torsdag and LA cry-s (DELLA1 della2), la cry-s (della1 della2) mutants in comparison with inoculated wild type in 9 days after inoculation (9 dai). The expression was normalized against the constitutively expressed ubiquitin gene. Panels $\mathbf{A}$ and $\mathbf{B}$ represent genes with various level of expression. For each gene, the transcript level in non-inoculated roots of wild type or mutants was set to 1 (control), and the level in inoculated wild type or mutants was calculated relative to the control values. Data are averages \pm SEM ( $n=6-8$ plants of wild type or mutant combined from three independent experiments). Values with different letters are significantly different $(P<0.05)$ as analyzed by one-way ANOVA and the Tukey's test as post-hoc analysis.

a significant reduction of expression of GA biosynthesis (PsGA20ox1) gene, but stimulation of GA deactivation (PsGA20x1) gene were found in non-inoculated roots of 6 days-old seedlings of la cry-s mutant compared with wild type (Weston et al., 2008). To estimate the expression dynamics of GA metabolic genes during nodulation, the transcription levels of the PsGA20ox1 and PsGA2ox1 genes have been measured in wild type and la cry-s mutant in 2, 9, and 14 dai using real-time PCR (Figure 5). We found a strong reduction in expression of the GA biosynthesis gene PsGA20ox1 at all stages in la cry-s mutant compared to wild type, but the most significant differences were found at early stages ( 2 and 9 dai). At the same time in la cry-s a strong stimulation of the GA deactivation gene PsGA2ox1 was found starting from 2 dai and reaching the highest level at 9 dai. This suggests that significant changes in GA metabolism may take place at initial stages of symbiosis development. It could not be excluded that the DELLA proteins are involved in this regulation, since previous studies have shown an important role of DELLA proteins in establishment of GA homeostasis (Zentella et al., 2007).

\section{Analysis of PsDELLA1 Protein Interaction With PsKNOX3, PsBELL1 in Yeast Two-Hybrid System (Y2H)}

To determine a possible interaction between pea DELLA1 protein and the KNOX and BELL transcription factors, we performed a yeast two-hybrid $(\mathrm{Y} 2 \mathrm{H})$ assay with the fusion proteins. Since the most significant role may play PsKNOX3 and PsBELL1 transcription factors, the analysis was performed with PsDELLA1-GAL4-DNA-binding domain (PsDELLA1-GAL4-DBD) and PsKNOX3-GAL4 activation 

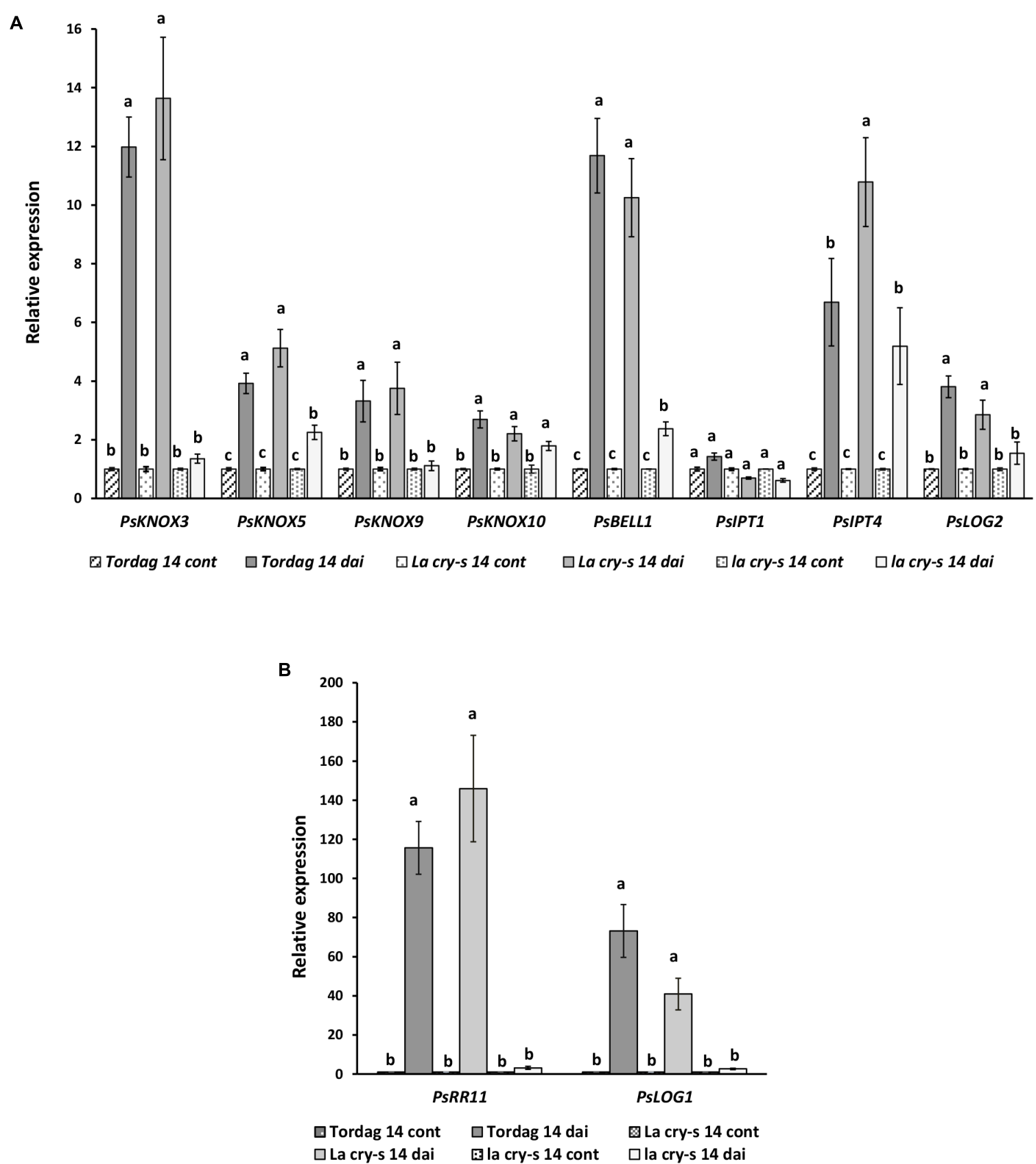

FIGURE 4 | Expression levels of PSKNOX and PSBELL genes and cytokinin metabolic genes in pea wild type cv. Torsdag and LA cry-s (DELLA1 della2), la cry-s (della1 della2) mutants in comparison with inoculated wild type in 14 days after inoculation (14 dai). Panels $\mathbf{A}$ and $\mathbf{B}$ represent genes with various level of expression. For each gene, the transcript level in non-inoculated roots of wild type or mutants was set to 1 (control), and the level in inoculated wild type or mutants was calculated relative to the control values. Data are averages \pm SEM $(n=4-6$ plants of wild type or mutant combined from two independent experiments). Values with different letters are significantly different $(P<0.05)$ as analyzed by one-way ANOVA and the Tukey's test as post-hoc analysis.

domain (PsKNOX3-GAL4-AD) as well as PsBELL1-GAL4 activation domain (PsBELL1-GAL4-AD).

Previously the capacity of DELLA proteins to promote CCaMK-IPD3/CYCLOPS and NSP2-NSP1 complexes formation was shown for model legumes Lotus japonicus and M. truncatula (Fonouni-Farde et al., 2016; Jin et al., 2016). To verify our results, we have also performed the analysis for PsDELLA1-GAL4-DNA-binding domain (PsDELLA1GAL4-DBD) and PsNSP2-GAL4 activation domain (PsNSP2-GAL4-AD), PsIPD3/CYCLOPS-GAL4 activation domain (PsIPD3/CYCLOPS-GAL4-AD). Indeed, the analysis of yeast growth on selection media showed that PsDELLA1 was able to form complex with PSNSP2 (SYM7) as well as PsIPD3/CYCLOPS (SYM33) in Y2H (Figures 6, 7). These 

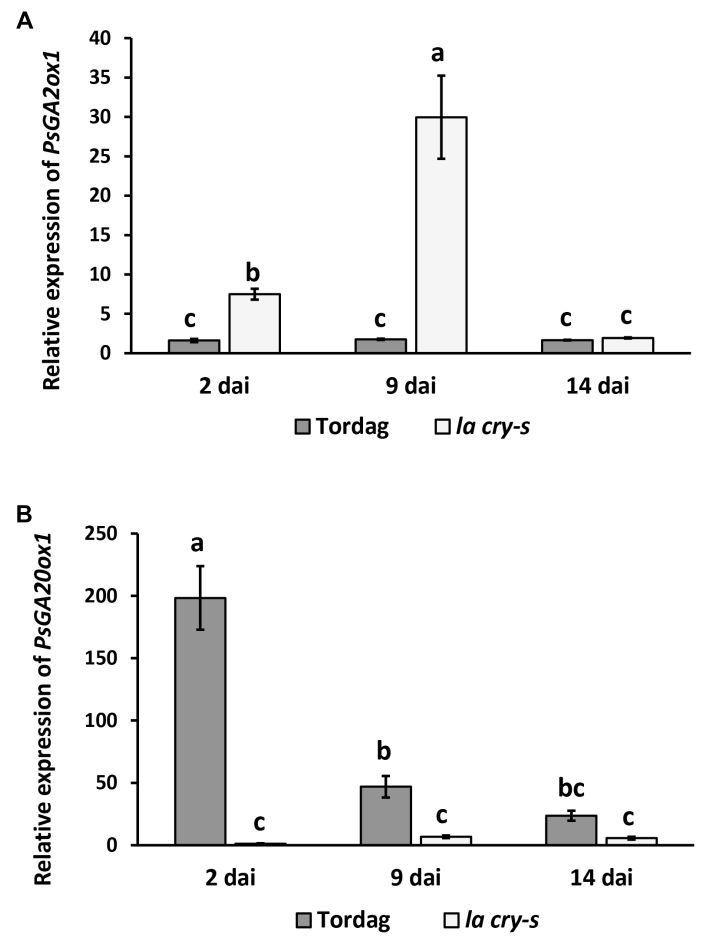

FIGURE 5 | PSGA20x1 (A) and PsGA200x1 (B) gene expression in roots of wild-type cv. Torsdag and la cry-s (della1 della 2) mutant plants inoculated by $R$. leguminosarum bv. viciae 3841 in 14 dai. For each gene, the transcript level in non-inoculated roots of wild type or mutants was set to 1 (control), and the level in inoculated wild type or mutants was calculated relative to the control values. Finally, the expression in wild type was presented relative to the level in mutant. Data are averages \pm SEM ( $n=4-8$ plants of wild type or mutant combined from two-three independent experiments). Values with different letters are significantly different $(P<0.05)$ as analyzed by one-way ANOVA and the Tukey's test as post-hoc analysis.

results were in accordance with results for $L$. japonicus and M. truncatula.

At the same time we did not reveal a strong interaction between PsDELLA1 and PsKNOX3, PsDELLA1 and PsBELL1 in this system (Figure 6). This conclusion can be made on the basis of the growth of MaV203 yeast cells producing both PsDELLA1GAL4-DBD and PsKNOX3-GAL4-AD, as well as PsDELLA1GAL4-DBD and PsBELL1-GAL4-AD on SC -LTH + $50 \mathrm{mM} 3$ AT, but not on SC - LTU. Therefore, we have not received the evidences of a strong direct interaction between PsDELLA1 and PsKNOX3 or PsBELL1 proteins.

\section{The Interplay Between DELLA, KNOX3, and IPD3/CYCLOPS Regulators}

Since DELLA appeared to be important in the regulation of PsKNOX3 and PsBELL1 expression in our experiments, we suggested that other transcription factors may be involved in transactivation of PsKNOX3 and PsBELL1 in pea. Among of them, the PsNSP2 and PsIPD3/CYCLOPS transcription factors are the most probable candidates for indirect activation of PsKNOX and PsBELL genes. Moreover, the interaction between
PsDELLA1 and PsNSP2, PsIPD3/CYCLOPS was shown in our experiments using $\mathrm{Y} 2 \mathrm{H}$ system.

Previously a similar effect was found for $M t E R N 1$ transcription factor, which is activated by MtDELLA1 through $M t \mathrm{NSP} 2 / M t \mathrm{NSP} 1$ complex formation and binding with promoter of MtERN1 (Fonouni-Farde et al., 2016). The binding with promoter induced the expression of MtERN1 and its target the MtENOD11 gene in M. truncatula.

Several pea mutants impaired in $n s p 2$ (sym7) and ipd3/cyclops (sym33) genes are available. The pea mutants E69 and RisNod14 are impaired in $n s p 2(s y m 7)$ gene and demonstrates the $\mathrm{Nod}^{-}$ phenotype (Engvild, 1987; Kneen et al., 1994; Walker and Downie, 2000; Tsyganov et al., 2002; Kalo et al., 2005). The pea mutants SGEFix ${ }^{-}-2$ and SGEFix $^{-}-5$ are impaired in ipd3/cyclops (sym33) gene ( $\mathrm{Fix}^{-}$phenotype) and able to form rare nodules which remained uninfected (Tsyganov et al., 1998; Voroshilova et al., 2009).

To check the interaction between PsDELLA1 protein and truncated PsNSP2 proteins corresponding to those in E69 and RisNod14 mutants as well as truncated PsIPD3/CYCLOPS protein corresponding to that in $\mathrm{SGEFix}^{-}-5$ we performed analysis using $\mathrm{Y} 2 \mathrm{H}$ system. This revealed that both truncated $P s$ NSP2 proteins were still able to interact with PsDELLA1, but truncated PsIPD3/CYCLOPS failed to interact with PsDELLA1 (Figure 7). This demonstrates that interruption of mutual recognition between PsDELLA1 and PsIPD3/CYCLOPS may result in signal transduction failure in $i p d 3 / c y c l o p s$ pea mutant. In case of two nsp2 (sym7) mutants the recognition between PsDELLA1 and PsNSP2 still takes place, but seems like the both truncated PsNSP2 are not able to interact with $P s N S P 1$ or other transcription factors and subsequent signal transduction is blocked.

Similarly, with la cry-s (della1 della2) mutant, a reduced number of nodules was found in ipd3/cyclops (sym33) mutants, although the nodules of ipd3/cyclops (sym33) were not effective ( Tsyganov et al., 1998; Ovchinnikova et al., 2011). Taking this into account, we might expect that in ipd3/cyclops (sym33) mutants the expression of PsKNOX3 and PsKNOX9 genes may be misregulated during infection as observed in la cry-s (della1 della2) mutant. To check this, we analyzed the expression of PsKNOX3, PsKNOX5, PsKNOX9, PsKNOX10, and PsBELL1 in roots bearing nodules (14 dai) from pea mutants SGEFix ${ }^{-}-5$ and SGEFix ${ }^{-}-2$ [ipd3/cyclops (sym33)]. Indeed, the SGEFix ${ }^{-}-5$ and SGEFix ${ }^{-}-2$ [ipd3/cyclops (sym33)] did not upregulated the expression of these genes as significantly as found in wild type plants (Figure 8). Our results suggest that the induction of $P_{s} K N O X 3, P_{s} K N O X 9$, and $P_{s} B E L L 1$ expression may require DELLA-dependent activation and analysis of ipd3/cyclops (sym33) mutants suggests this may be via interaction with PsIPD3/CYCLOPS transcription factor. In addition, a reduced transcription level of the GA biosynthesis gene PsGA20ox1 was found in ipd3/cyclops (sym33) mutants compared to wild type in 14 dai using real-time PCR. Decreased level of PsGA20ox1 expression in della1 della2 and ipd3/cyclops (sym33) mutants may reflect the importance of GA for nodule development in pea. Upregulation of GA metabolic genes may be also connected with activation of $P_{s} \mathrm{KNOX}$ and $P_{s} \mathrm{BELL}$ transcription factors. 


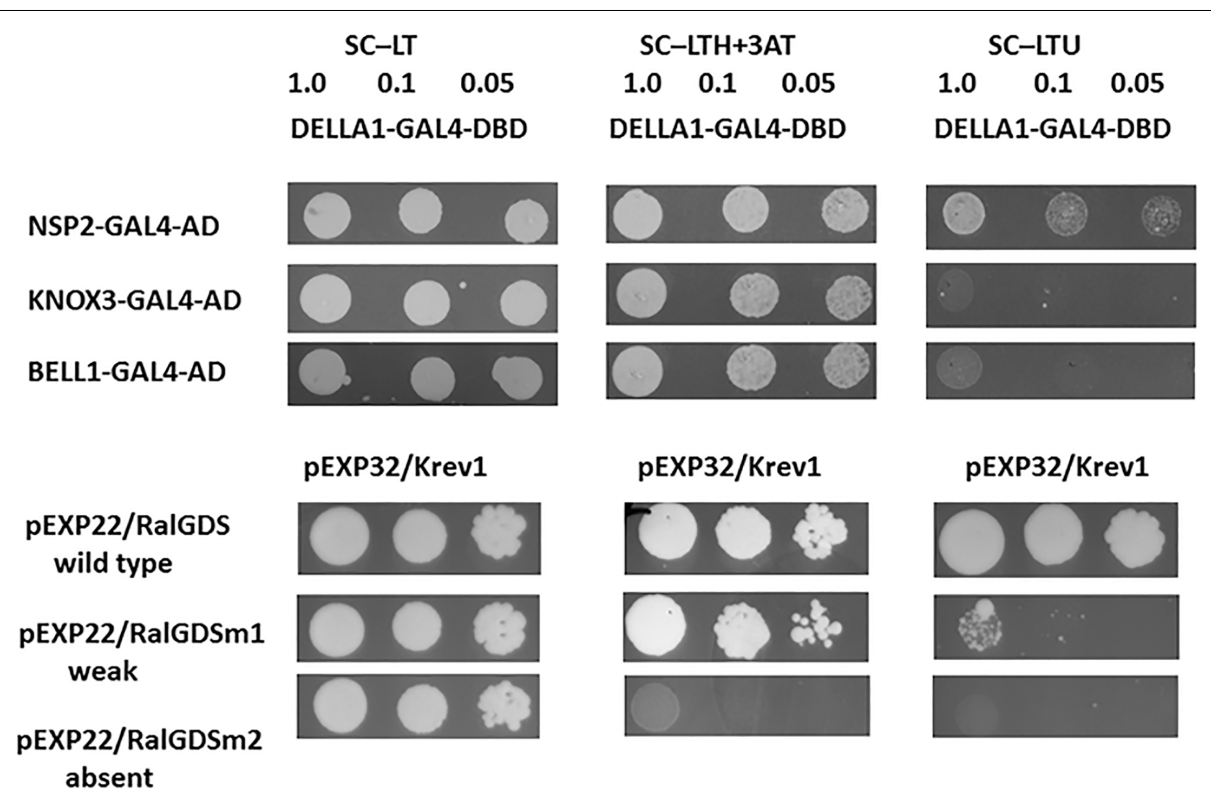

FIGURE 6 | Yeast two-hybrid assay (GAL4 transcription factor-based) for DELLA1 protein and KNOX3 and BELL1 transcription factors. The S. cerevisiae strain MaV203 was transformed simultaneously with pDEST22 and pDEST32 vectors for GAL4 activation domain (AD) or GAL4 DNA binding domain (DBD). The pairs of vectors pEXP32/Krev1 and pEXP22/RalGDS-wild type, pEXP22/RalGDS-m1 and pEXP22/RalGDS-m2 were used for strong, weak and not detectable interactions. Yeast growth was tested on SC medium without leucine, tryptophan and histidine with 3-amino-triazole (SC -LTH + 50 mM 3 AT) and SC without leucine, tryptophan and uracil (SC - LTU).

\section{DISCUSSION}

Multiple studies have shown an important role of CKs in regulation of infection process and nodule organogenesis during legume-rhizobial symbiosis. In addition to CKs, the GAs also have a strong impact on symbiosis development. Indeed, transcriptome analyses revealed the substantial alterations in the expression of genes encoding enzymes of CK and GA biosynthesis and degradation during symbiosis initiation and development in legume plants (Breakspear et al., 2014; van Zeijl et al., 2015; Jardinaud et al., 2016). The antagonistic relationships between CKs and GAs were shown in many developmental processes and were also described in nodulation in M. truncatula and L. japonicus (Maekawa et al., 2009; Jin et al., 2016). It was shown that the number of spontaneous nodules induced by overexpression of LjLHK1/MtCRE1 genes encoding the receptor to CKs was decreased upon the addition of GAs (Maekawa et al., 2009; Jin et al., 2016). This effect was depended on DELLA proteins, suggesting that DELLAs were required for spontaneous nodule formation induced by CKs, but GAs inhibited this process.

Recent study has also revealed that GA pretreatment reduced the Nod factor-induced CK primary response in the epidermis and in the outer cortex in M. truncatula as well as CK response gene expression, the MtRRA8, MtRRA9, MtRRA11 (FonouniFarde et al., 2017). It has been also shown that pretreatment with GAs may decrease the amount of some pools of bioactive CKs in roots in M. truncatula (Fonouni-Farde et al., 2017).

Indeed, exogenous GAs has a negative effect on the number of nodules in M. truncatula and L. japonicus and this was also seen at high concentrations of GA in pea (Ferguson, 2005; Maekawa et al., 2009; Fonouni-Farde et al., 2016; Jin et al., 2016). However, at lower concetrations GA actually enhanced nodule number in pea and Sesbania (Ferguson, 2005; Lievens et al., 2005). This indicates a more complicated role for GAs during nodulation. While this apparent paradox may be explained by stating that there is an optimal level of GAs for nodulation overall, this may in fact be due to GAs exerting different effects on specific stages of nodulation, inhibiting infection but promoting nodule organogenesis (Ferguson, 2005; Ferguson et al., 2011; McAdam et al., 2018). This is similar to the dual role proposed for CKs during nodulation (Gamas et al., 2017).

This regulation could be achieved by interaction between DELLA proteins and plant regulators controlling the content of CKs and GAs in plants. Since the participation of KNOX and BELL transcription factors in regulation of CK and GA metabolic genes is well-known for plants, the pea la cry$s$ (della1 della2) mutants have been used to examine the effect of these proteins on the expression of PSKNOX and PsBELL genes during nodulation. Indeed, we showed failure to upregulate the expression of PsKNOX9 and PsBELL1 genes at early stages of symbiosis development in inoculated roots of pea la cry-s (della1 della2) mutant. Although we did not find a definite correlation between PsDELLAs function and regulation of some PSIPT genes at early stages, but influence on other CK metabolic genes could not be excluded. Recently the involvement of GAs in stimulation of CKs degradation via cytokinin oxidase $M t \mathrm{CKX} 3$ has been revealed in M. truncatula (Fonouni-Farde et al., 2017). An alternative explanation might 


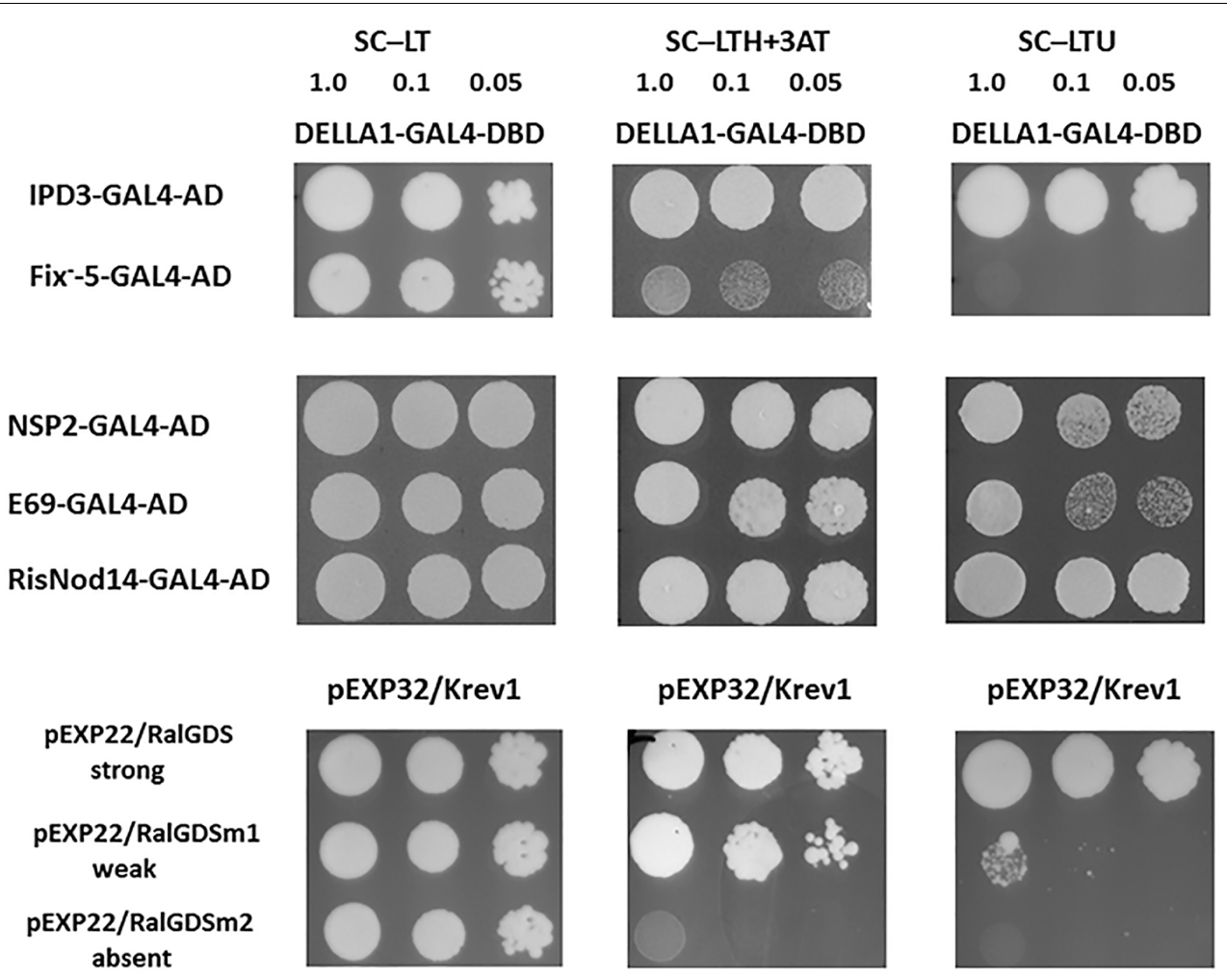

FIGURE 7 | Yeast two-hybrid assay (GAL4 transcription factor-based) for DELLA1 protein and NSP2 and IPD3/CYCLOPS transcription factors and their truncated variants corresponding to those in E69 (nsp2/sym7-1), RisNOD14 (nsp2/sym7-2) and SGEFix ${ }^{-}-5$ (ipd3/cyclops/sym33-2). AD - activation domain of GAL4, DBD DNA binding domain of GAL4. The pairs of vectors pEXP32/Krev1 and pEXP22/RalGDS-wild type, pEXP22/RalGDS-m1 and pEXP22/RalGDS-m2 were used for strong, weak and not detectable interactions. Yeast growth was tested on SC medium -LTH + $50 \mathrm{mM} 3$ AT and SC - LTU.

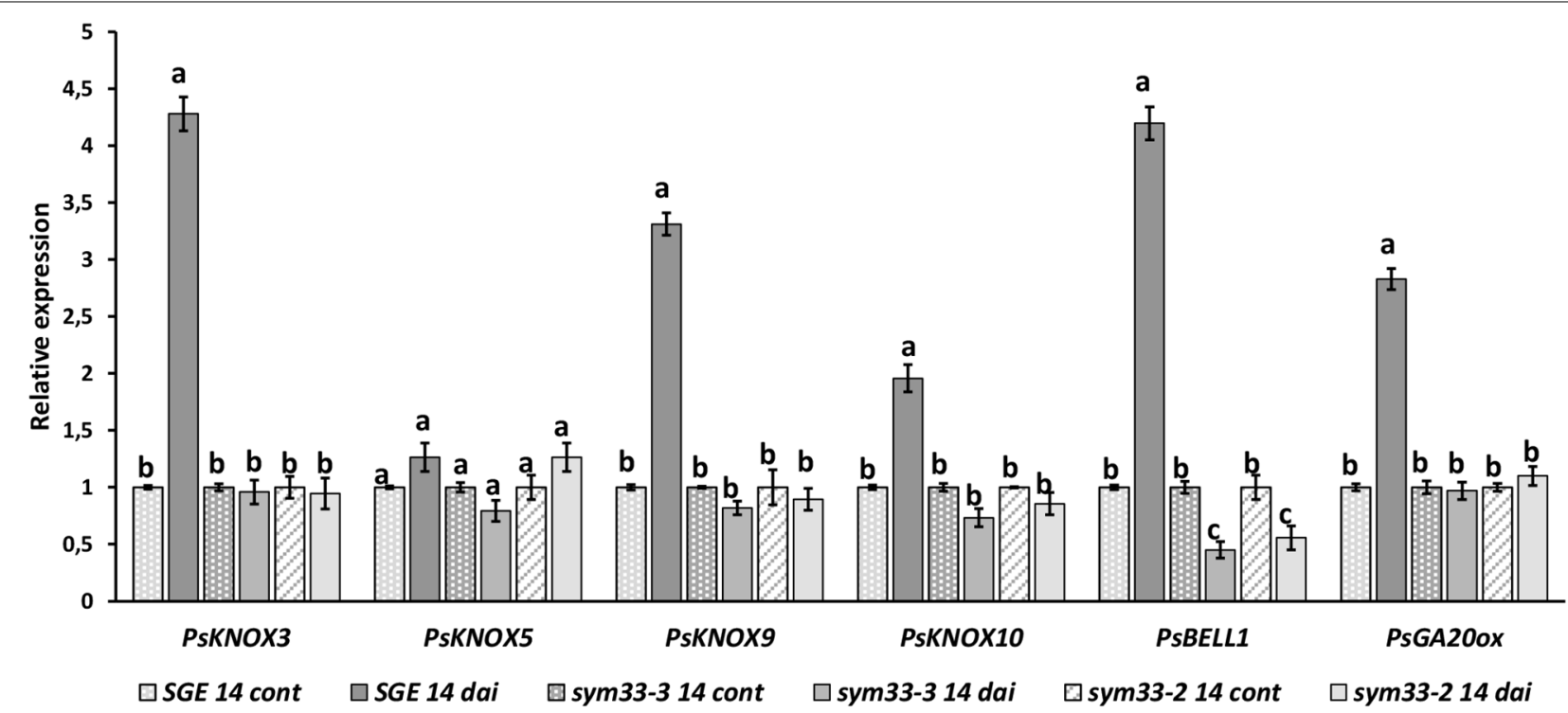

FIGURE 8| Expression analysis of PSGA20ox, PSKNOX, and PSBELL genes in wild type cv. SGE and SGEFix ${ }^{-}-2$ (sym33-3), SGEFix ${ }^{-}-5$ (sym33-2) mutants 2 weeks after inoculation. Transcript levels of genes were normalized against ubiquitin gene. For each gene, the transcript level in non-inoculated roots of wild type or mutants was set to 1 (control), and the level in nodules of wild type or mutants was calculated relative to the control values. Data are averages \pm SEM ( $n=6-8$ plants of wild type or mutant combined from two independent experiments). Values with different letters are significantly different $(P<0.05)$ as analyzed by one-way ANOVA and the Tukey's test as post-hoc analysis. 
be that PsKNOX9 and PsBELL1 influence GA metabolic genes at early stages of symbiosis, as revealed significant changes in GA metabolism may take place at initial stages of symbiosis development and PsDELLA proteins are involved in this regulation.

At later stages of symbiosis development the up regulation of expression of PsKNOX3, PsKNOX9, and PsBELL1 was shown to be attenuated in pea della1 della2 mutant. However, up regulation in the expression of other PsKNOX genes, the PsKNOX5 and PsKNOX10, was only slightly reduced or similar in della mutant compared to wild type. Consistently, pea la cry$s$ (della1 della2) mutant showed reduced upregulation in the expression of the CK synthesis (PsLOG1, PsLOG2) and signaling (PsRR11) genes during nodulation compared to wild type. One interpretation is that PsDELLA proteins can regulate the CK metabolism via PsKNOX and PsBELL transcription factors during nodule development. Upregulation of CK metabolic genes has previously been shown to be due to activation of KNOX and BELL transcription factors (Yanai et al., 2005; Azarakhsh et al., 2015).

As has been shown in analysis of model legumes, the DELLA proteins may regulate a number of transcription factors during nodulation. In some cases the DELLA proteins interact with individual transcription factors including NSP2, IPD3/CYCLOPS and NF-YA1 (direct activation), in other cases their effect may be indirect. Since DELLAs don't have a DNA-binding motif, they may induce binding of activated transcription factors with promoter of genes encoding other transcription factors like ERN1 (Fonouni-Farde et al., 2016).

To verify the effect of PsDELLA proteins on PsKNOX3 and PsBELL1 transcription factors, the possibility of their interaction has been investigated using $\mathrm{Y} 2 \mathrm{H}$ system. However, our studies did not reveal a strong direct interaction between PsDELLA1 and PsKNOX3 or PsBELL1 in this heterologous system. We suggested that PsDELLA1 may promote the expression PsKNOX3 and PsBELL1, probably through other known transcription factors. This function may be related to IPD3/CYCLOPS and NSP2 transcription factors, because DELLA proteins can activate them during nodulation (Floss et al., 2016; Fonouni-Farde et al., 2016; Jin et al., 2016). We tested the interaction between PsDELLA1 and truncated proteins corresponding to those in pea ipd3/cyclops (sym33) and nsp2 (sym7) mutants. Surprisingly, only PsIPD3/CYCLOPS truncated protein failed to interact with PsDELLA1, but PsDELLA1 still interacted with both PsNSP2 truncated proteins. It suggests that in both ipd3/cyclops (sym33) mutants the interaction between PsDELLA1 and PsIPD3/CYCLOPS may be critical for subsequent signal transduction. Since similar trends for reduced ability to up regulate the expression of PsKNOX3 and PsKNOX9 transcription levels were found in the nodules of ipd3/cyclops (sym33) mutants as seen in della mutants compared with wild type, the PsDELLA proteins may promote the expression of PsKNOX3 and PsBELL1 genes via the PsIPD3/CYCLOPS transcription factor. Additional experiments that could explore this further include analyzing the binding capacity of PsIPD3/CYCLOPS with promoters of the PsKNOX3 and
PsKNOX9 genes in pea plants and the subsequent effect on target genes.

As previously reported (Ferguson et al., 2011), la cry-s mutant had significantly fewer nodules than wild-type plants. Similarly, ipd3/cyclops (sym33) mutants had a considerably reduced number of nodules (Tsyganov et al., 1998; Ovchinnikova et al., 2011). Therefore, it may suggest the involvement of PsDELLA proteins and PsIPD3/CYCLOPS transcription factor in initiation of nodule organogenesis and regulation of nodule number in pea plants. As it was shown for model legume plants $M$. truncatula and L. japonicus, the DELLA proteins are involved in activation of IPD3/CYCLOPS transcription factor. Our findings revealed that DELLA proteins and IPD3/CYCLOPS also influence the expression of pea $K N O X$ and BELL genes encoding transcription factors.

The infection thread formation was significantly reduced in DELLA-deficient pea la cry-s double mutants compared with wild-type plants (McAdam et al., 2018). Similar to la cry-s, pea ipd3/cyclops (sym33) also showed impaired invasion of the nodule primordia at early stages (Voroshilova et al., 2009). The most probable explanation that impaired infection may influence the signal exchange between epidermis and cortical cells resulting in decreased number of nodule primordia in both type of mutants. Indeed, the experiments in which the expression of GA-insensitive MtDELLA1 was restricted by epidermis showed that it was able to trigger the spontaneous nodule formation in spatially distant cortical cells in absence of rhizobia (Fonouni-Farde et al., 2017). It suggests that some diffusive factors may be involved in signal transduction in this case. Since the KNOX and BELL transcription factors may act in non-cell autonomous manner, additional experiments should explore a possible role of KNOX and BELL as such diffusive factors.

\section{AUTHOR CONTRIBUTIONS}

$\mathrm{AD}$ and $\mathrm{ED}$ conceived the study and designed the experiments. $\mathrm{AD}$ and $\mathrm{AK}$ performed the experiments and analyzed the data. IT supervised. $\mathrm{AD}, \mathrm{EF}$, and $\mathrm{ED}$ wrote the manuscript.

\section{FUNDING}

This work was financially supported by the Russian Science Foundation (Grant 17-76-30016). The research was performed using equipment of the Core Centrum "Genomic Technologies, Proteomics and Cell Biology" in ARRIAM.

\section{SUPPLEMENTARY MATERIAL}

The Supplementary Material for this article can be found online at: https://www.frontiersin.org/articles/10.3389/fpls.2019.00432/ full\#supplementary-material 


\section{REFERENCES}

Achard, P., Baghour, M., Chapple, A., Hedden, P., Van Der Straeten, D., Genschik, P., et al. (2007). The plant stress hormone ethylene controls floral transition via DELLA-dependent regulation of floral meristem-identity genes. Proc. Natl. Acad. Sci. U.S.A. 104, 6484-6489. doi: 10.1073/pnas.0610717104

Achard, P., Cheng, H., De Grauwe, L., Decat, J., Schoutteten, H., Moritz, T., et al. (2006). Integration of plant responses to environmentally activated phytohormonal signals. Science 311, 91-94. doi: 10.1126/science.1118642

Alvey, L., and Harberd, N. P. (2005). DELLA proteins: integrators of multiple plant growth regulatory inputs? Physiol. Plant. 123, 153-160. doi: 10.1111/j.13993054.2004.00412.x

Azarakhsh, M., Kirienko, A. N., Zhukov, V. A., Lebedeva, M. A., Dolgikh, E. A., and Lutova, L. A. (2015). KNOTTED1-LIKE HOMEOBOX 3: a new regulator of symbiotic nodule development. J. Exp. Bot. 66, 7181-7195. doi: 10.1093/jxb/ erv414

Bao, X., Franks, R. G., Levin, J. Z., Liu, Z., The, S., Cell, P., et al. (2004). Repression of AGAMOUS by BELLRINGER in floral and inflorescence meristems. Plant Cell 16, 1478-1489. doi: 10.1105/tpc.021147

Bellaoui, M., Pidkowich, M. S., Samach, A., Kushalappa, K., Kohalmi, S. E., Modrusan, Z., et al. (2001). The Arabidopsis BELL1 and KNOX TALE homeodomain proteins interact through a domain conserved between plants and animals. Plant Cell 13, 2455-2470. doi: 10.1105/tpc.010161

Breakspear, A., Liu, C., Roy, S., Stacey, N., Rogers, C., Trick, M., et al. (2014). The root hair "Infectome" of Medicago truncatula uncovers changes in cell cycle genes and reveals a requirement for auxin signaling in rhizobial infection. Plant Cell 26, 4680-4701. doi: 10.1105/tpc.114.133496

Brian, P. W. (1959). Effects of gibberellins on plant growth and development. Biol. Rev. 34, 37-77. doi: 10.1111/j.1469-185X.1959.tb01301.x

Byrne, M. E., Kidner, C. A., and Martienssen, R. A. (2003). Plant stem cells: divergent pathways and common themes in shoots and roots. Curr. Opin. Genet. Dev. 13, 551-557. doi: 10.1016/j.gde.2003.08.008

Chen, H., Banerjee, A. K., and Hannapel, D. J. (2004). The tandem complex of BEL and KNOX partners is required for transcriptional repression of ga20ox1. Plant J. 38, 276-284. doi: 10.1111/j.1365-313X.2004.02048.x

Cooper, J. B., and Long, S. R. (1994). Morphogenetic rescue of rhizobium meliloti nodulation mutants by trans-zeatin secretion. Plant Cell 6, 215-225. doi: 10. 1105/tpc.6.2.215

Davière, J. M., and Achard, P. (2013). Gibberellin signaling in plants. Development 140, 1147-1151. doi: 10.1242/dev.087650

Dolgikh, E. A., Shaposhnikov, A. I., Dolgikh, A. V., Gribchenko, E. S., Bodyagina, K. B., Yuzhikhin, O. S., et al. (2017). Identification of Pisum sativum L. cytokinin and auxin metabolic and signaling genes, and an analysis of their role in symbiotic nodule development. Int. J. Plant Physiol. Biochem. 9, 22-35. doi: 10.5897/IJPPB2017.0266

Engvild, K. C. (1987). Nodulation and nitrogen fixation mutants of pea, Pisum sativum. Theor. Appl. Genet. 74, 711-713. doi: 10.1007/BF00247546

Ferguson, B. J. (2005). Nodulation phenotypes of gibberellin and brassinosteroid mutants of pea. Plant Physiol. 138, 2396-2405. doi: 10.1104/pp.105.062414

Ferguson, B. J., Foo, E., Ross, J. J., and Reid, J. B. (2011). Relationship between gibberellin, ethylene and nodulation in Pisum sativum. New Phytol. 189, 829-842. doi: 10.1111/j.1469-8137.2010.03542.x

Ferguson, B. J., and Mathesius, U. (2014). Phytohormone regulation of legumerhizobia interactions. J. Chem. Ecol. 40, 770-790. doi: 10.1007/s10886-0140472-7

Fleet, C. M., and Sun, T. P. (2005). A DELLAcate balance: the role of gibberellin in plant morphogenesis. Curr. Opin. Plant Biol. 8, 77-85. doi: 10.1016/j.pbi.2004. 11.015

Floss, D. S., Lévesque-Tremblay, V., Park, H. J., and Harrison, M. J. (2016). DELLA proteins regulate expression of a subset of AM symbiosis-induced genes in medicago truncatula. Plant Signal. Behav. 11:e1162369. doi: 10.1080/15592324. 2016.1162369

Fonouni-Farde, C., Kisiala, A., Brault, M., Emery, R. J. N., Diet, A., and Frugier, F. (2017). DELLA1-mediated gibberellin signaling regulates cytokinin-dependent symbiotic nodulation. Plant Physiol. 175, 1795-1806. doi: 10.1104/pp.17.00919

Fonouni-Farde, C., Tan, S., Baudin, M., Brault, M., Wen, J., Mysore, K. S., et al. (2016). DELLA-mediated gibberellin signalling regulates Nod factor signalling and rhizobial infection. Nat. Commun. 7:12636. doi: 10.1038/ncomms12636
Gamas, P., Brault, M., Jardinaud, M. F., and Frugier, F. (2017). Cytokinins in symbiotic nodulation: when, where, what for? Trends Plant Sci. 22, 792-802. doi: 10.1016/j.tplants.2017.06.012

Gonzalez-Rizzo, S., Crespi, M., and Frugier, F. (2006). The Medicago truncatula CRE1 cytokinin receptor regulates lateral root development and early symbiotic interaction with Sinorhizobium meliloti. Plant Cell 18, 2680-2693. doi: 10.1105/ tpc. 106.043778

Hake, S., Smith, H. M. S., Holtan, H., Magnani, E., Mele, G., and Ramirez, J. (2004). The role of KNOX genes in plant development. Annu. Rev. Cell Dev. Biol. 20, 125-151. doi: 10.1146/annurev.cellbio.20.031803.093824

Hall, B. G. (2013). Building phylogenetic trees from molecular data with MEGA. Mol. Biol. Evol. 30, 1229-1235. doi: 10.1093/molbev/mst012

Harberd, N. P., King, K. E., Carol, P., Cowling, R. J., Peng, J., and Richards, D. E. (1998). Gibberellin: inhibitor of an inhibitor of.?. BioEssays 20, 1001-1008. doi: 10.1002/(SICI)1521-1878(199812)20:12<1001::AID-BIES6<3.0.CO;2-O

Heckmann, A. B., Sandal, N., Bek, A. S., Madsen, L. H., Jurkiewicz, A., Nielsen, M. W., et al. (2011). Is regulated by a mechanism operating in the root cortex. Mol. Plant-Microbe Interact. 24, 1385-1395. doi: 10.1094/MPMI-05-11-0142

Hedden, P., and Thomas, S. G. (2012). Gibberellin biosynthesis and its regulation. Biochem. J. 444, 11-25. doi: 10.1042/BJ20120245

Hofer, J., Gourlay, C., Michael, A., and Ellis, T. H. N. (2001). Expression of a class 1 knotted1-like homeobox gene is down-regulated in pea compound leaf primordia. Plant Mol. Biol. 45, 387-398. doi: 10.1023/A:1010739812836

Jardinaud, M.-F., Boivin, S., Rodde, N., Catrice, O., Kisiala, A., Lepage, A., et al. (2016). A laser dissection-RNAseq analysis highlights the activation of cytokinin pathways by nod factors in the Medicago truncatula root epidermis. Plant Physiol. 171, 2256-2276. doi: 10.1104/pp.16.00711

Jasinski, S., Piazza, P., Craft, J., Hay, A., Woolley, L., Rieu, I., et al. (2005). KNOX action in Arabidopsis is mediated by coordinate regulation of cytokinin and gibberellin activities. Curr. Biol. 15, 1560-1565. doi: 10.1016/j.cub.2005.07.023

Jin, Y., Liu, H., Luo, D., Yu, N., Dong, W., Wang, C., et al. (2016). DELLA proteins are common components of symbiotic rhizobial and mycorrhizal signalling pathways. Nat. Commun. 7, 1-14. doi: 10.1038/ncomms12433

Kalo, P., Gleason, C., Edwards, A., Marsh, J., Mitra, R. M., and Hirsch, S. (2005). Nodulation signaling in legumes requires NSP2, a member of the GRAS family of transcriptional regulators. Science 308, 1786-1789. doi: 10.1126/science. 1110951

Kneen, B. E., Weeden, N. F., and Larue, T. A. (1994). Non-nodulating mutants of Pisum Sativum (L.) cv. sparkle. J. Hered. 85, 129-133. doi: 10.1093/ oxfordjournals.jhered.a111410 doi: 10.1093/oxfordjournals.jhered.a111410

Lievens, S., Goormachtig, S., Den Herder, J., Capoen, W., Mathis, R., and Hedden, P. (2005). Gibberellins are involved in nodulation of Sesbania rostrata. Plant Physiol. 139, 1366-1379. doi: 10.1104/pp.105.066944

Lin, T., Sharma, P., Gonzalez, D. H., Viola, I. L., and Hannapel, D. J. (2013). The impact of the long-distance transport of a BEL1-like messenger RNA on development. Plant Physiol. 161, 760-772. doi: 10.1104/pp.112.209429

Livak, K. J., and Schmittgen, T. D. (2001). Analysis of relative gene expression data using real-time quantitative PCR and the $2^{-\Delta \Delta \mathrm{Ct}}$ method. Methods 25, 402-408. doi: 10.1006/meth.2001.1262

Maekawa, T., Maekawa-Yoshikawa, M., Takeda, N., Imaizumi-Anraku, H., Murooka, Y., and Hayashi, M. (2009). Gibberellin controls the nodulation signaling pathway in Lotus japonicus. Plant J. 58, 183-194. doi: 10.1111/j.1365313X.2008.03774.x

McAdam, E. L., Reid, J. B., and Foo, E. (2018). Gibberellins promote nodule organogenesis but inhibit the infection stages of nodulation. J. Exp. Bot. 69, 2117-2130. doi: 10.1093/jxb/ery046

Murray, J., Karas, B., Ross, L., Brachmann, A., Wagg, C., Geil, R., et al. (2006). Genetic suppressors of the Lotus japonicus har1-1 hypernodulation phenotype. Mol Plant-Microbe Interact. 19, 1082-1091. doi: 10.1094/MPMI19-1082

Navarro, L., Bari, R., Achard, P., Lisón, P., Nemri, A., Harberd, N. P., et al. (2008). DELLAs control plant immune responses by modulating the balance of jasmonic acid and salicylic acid signaling. Curr. Biol. 18, 650-655. doi: 10. 1016/j.cub.2008.03.060

Ovchinnikova, E., Journet, E.-P., Chabaud, M., Cosson, V., Ratet, P., Duc, G., et al. (2011). IPD3 controls the formation of nitrogen-fixing symbiosomes in pea and Medicago Spp. Mol. Plant-Microbe Interact. 24, 1333-1344. doi: 10.1094/ MPMI-01-11-0013 
Park, J., Nguyen, K. T., Park, E., Jeon, J.-S., and Choi, G. (2013). DELLA proteins and their interacting RING finger proteins repress gibberellin responses by binding to the promoters of a subset of gibberellin-responsive genes in Arabidopsis. Plant Cell 25, 927-943. doi: 10.1105/tpc.112.108951

Plet, J., Wasson, A., Ariel, F., Le Signor, C., Baker, D., Mathesius, U., et al. (2011). MtCRE1-dependent cytokinin signaling integrates bacterial and plant cues to coordinate symbiotic nodule organogenesis in Medicago truncatula. Plant J. 65, 622-633. doi: 10.1111/j.1365-313X.2010.04447.x

Sharma, P., Lin, T., Grandellis, C., Yu, M., and Hannapel, D. J. (2014). The BEL1like family of transcription factors in potato. J. Exp. Bot. 65, 709-723. doi: $10.1093 /$ jxb/ert432

Smith, H. M. S., and Hake, S. (2003). The interaction of two homeobox genes. Plant Cell 15, 1717-1727. doi: 10.1105/tpc.012856.stems

Sun, T., and Gubler, F. (2004). Molecular mechanism of gibberellin signaling in plants. Annu. Rev. Plant Biol. 55, 197-223. doi: 10.1146/annurev.arplant.55. 031903.141753

Sun, T. P. (2010). Gibberellin-GID1-DELLA: a pivotal regulatory module for plant growth and development. Plant Physiol. 154, 567-570. doi: 10.1104/pp.110. 161554

Thompson, J. D., Higgins, D. G., and Gibson, T. J. (1994). CLUSTAL W: improving the sensitivity of progressive multiple sequence alignment through sequence weighting, position-specific gap penalties and weight matrix choice. Nucleic Acids Res. 22, 4673-4680. doi: 10.1093/nar/22.22. 4673

Tirichine, L., Sandal, N., Madsen, L. H., Radutoiu, S., Albrektsen, A. S., Sato, S., et al. (2007). A gain-of-function mutation in a root nodule organogenesis. Science 2680, 104-107. doi: 10.1126/science.1132397

Tsyganov, V. E., Morzhina, E. V., Stefanov, S. Y., Borisov, A. Y., Lebsky, V. K., and Tikhonovich, I. A. (1998). The pea (Pisum sativum L.) genes sym 33 and sym 40 control infection thread formation and root nodule function. Mol. Gen. Genet. 259, 491-503. doi: 10.1007/s004380050840

Tsyganov, V. E., Voroshilova, V. A., Priefer, U. B., Borisov, A. Y., and Tikhonovich, I. A. (2002). Genetic dissection of the initiation of the infection process and nodule tissue development in the Rhizobium-pea (Pisum sativum L.) symbiosis. Ann. Bot. 89, 357-366. doi: 10.1093/aob/mcf051

van Brussel, A. A., Tak, T., Wetselaar, A., Pees, E., and Wijffelman, C. (1982). Small leguminosae as test plants for nodulation of Rhizobium leguminosarum and other rhizobia and agrobacteria harbouring a leguminosarum sym-plasmid. Plant Sci. Lett. 27, 317-325. doi: 10.1016/0304-4211(82) 90134-1 van Brussel, A. A. N., Planque, K., and Quispel, A. (1977). The wall of Rhizobium leguminosarum in bacteroid and free-living forms. J. Gen. Microbiol. 101, 51-56. doi: 10.1099/00221287-101-1-51

van Zeijl, A., Liu, W., Xiao, T. T., Kohlen, W., Yang, W. C., Bisseling, T., et al. (2015). The strigolactone biosynthesis gene DWARF27 is co-opted in rhizobium symbiosis. BMC Plant Biol. 15:260. doi: 10.1186/s12870-015-0651-x

Voroshilova, V. A., Demchenko, K. N., Brewin, N. J., Borisov, A. Y., and Tikhonovich, I. A. (2009). Initiation of a legume nodule with an indeterminate meristem involves proliferating host cells that harbour infection threads. New Phytol. 181, 913-923. doi: 10.1111/j.1469-8137.2008.02723.x

Walker, S. A., and Downie, J. A. (2000). Entry of Rhizobium leguminosarum bv. viciae into root hairs requires minimal nod factor specificity, but subsequent infection thread growth requires nodo or node. Mol. Plant-Microbe Interact. 13, 754-762. doi: 10.1094/MPMI.2000.13.7.754

Weston, D. E., Elliott, R. C., Lester, D. R., Rameau, C., Reid, J. B., Murfet, I. C., et al. (2008). The pea DELLA proteins LA and CRY are important regulators of gibberellin synthesis and root growth. Plant Physiol. 147, 199-205. doi: 10.1104/pp. 108.115808

Yanai, O., Shani, E., Dolezal, K., Tarkowski, P., Sablowski, R., Sandberg, G., et al. (2005). Arabidopsis KNOXI proteins activate cytokinin biosynthesis. Curr. Biol. 15, 1566-1571. doi: 10.1016/j.cub.2005.07.060

Zentella, R., Zhang, Z.-L., Park, M., Thomas, S. G., Endo, A., Murase, K., et al. (2007). Global analysis of DELLA direct targets in early gibberellin signaling in Arabidopsis. Plant Cell 19, 3037-3057. doi: 10.1105/tpc.107.054999

Zhou, C., Han, L., Li, G., Chai, M., Fu, C., Cheng, X., et al. (2014). STM/BPLike KNOXI is uncoupled from ARP in the regulation of compound leaf development in Medicago truncatula. Plant Cell 26, 1464-1479. doi: 10.1105/ tpc. 114.123885

Conflict of Interest Statement: The authors declare that the research was conducted in the absence of any commercial or financial relationships that could be construed as a potential conflict of interest.

Copyright (c) 2019 Dolgikh, Kirienko, Tikhonovich, Foo and Dolgikh. This is an open-access article distributed under the terms of the Creative Commons Attribution License (CC BY). The use, distribution or reproduction in other forums is permitted, provided the original author(s) and the copyright owner(s) are credited and that the original publication in this journal is cited, in accordance with accepted academic practice. No use, distribution or reproduction is permitted which does not comply with these terms. 